\title{
Transport and recombination through weakly coupled localized spin pairs in semiconductors during coherent spin excitation
}

\author{
V. Rajevac, ${ }^{1,2}$ C. Boehme, ${ }^{3}$ C. Michel, ${ }^{1}$ A. Gliesche,${ }^{4}$ K. Lips,${ }^{2}$ S. D. Baranovskii, ${ }^{1}$ and P. Thomas ${ }^{1}$ \\ ${ }^{1}$ Fachbereich Physik, Philipps-Universität-Marburg, D-35032 Marburg, Germany \\ ${ }^{2}$ Hahn-Meitner-Institut Berlin, Kekuléstrasse 5, D-12489 Berlin, Germany \\ ${ }^{3}$ Physics Department, University of Utah, 115S 1400E, Salt Lake City, Utah 84112, USA \\ ${ }^{4}$ Ecole Polytechnique Fédérale, Institut de Théorie des Phénomènes Physiques, CH-1015 EPF-Lausanne, Switzerland
}

(Received 5 July 2006; published 11 December 2006)

\begin{abstract}
Semianalytical predictions for the transients of spin-dependent transport and recombination rates through localized states in semiconductors during coherent electron-spin excitation are made for the case of weakly spin-coupled charge-carrier ensembles. The results show that the on-resonant Rabi frequency of electrically or optically detected spin oscillation doubles abruptly as the strength of the resonant microwave field $\gamma B_{1}$ exceeds the Larmor frequency separation within the pair of charge-carrier states between which the transport or recombination transition takes place. For the case of a Larmor frequency separation of the order of $\gamma B_{1}$ and arbitrary excitation frequencies, the charge carrier-pairs exhibit four different nutation frequencies. From the calculations, a simple set of equations for the prediction of these frequencies is derived.
\end{abstract}

DOI: 10.1103/PhysRevB.74.245206

PACS number(s): 72.20.-i, 76.30.-v, 76.70.Hb, 76.90.+d

\section{INTRODUCTION}

Electrically and optically detected magnetic resonance experiments (EDMR and ODMR, respectively) are alternative ways to detect electron-spin resonances (ESR) in materials with charge-carrier transport or recombination transitions that are governed by spin-selection rules. ${ }^{1-7}$ The advantage of EDMR and ODMR in comparison with the traditional ESR spectroscopy is the sensitivity of these methods, which is typically 6 to 10 orders of magnitude higher. ${ }^{3,4}$ This has become particularly useful for the investigation of paramagnetic centers in highly diluted matrices or low-dimensional semiconductor thin-film devices and interfaces, point defects, ${ }^{1,2,8-10}$ and defect clusters. One of the challenges of EDMR and ODMR spectroscopy is that the information obtained from these experiments is different in comparison to the ESR data. ${ }^{11}$ The reasons for the discrepancies between ESR and EDMR/ODMR are mainly due to the two different measurement approaches, which imply two different observables: When the density operator $\hat{\rho}$ represents the spin ensemble to be investigated, the observable corresponding to ESR experiments will always be spin polarization $\langle\vec{P}\rangle$ $=\operatorname{Tr}(\hat{\mathrm{P}} \hat{\rho})$ represented by the spin polarization operator $\hat{P}$, whereas for the indirect detection through spin-dependent transport or recombination, the observables are the permutation-symmetry or -antisymmetry operators represented by the singlet $|S\rangle\langle S|$ or triplet operators $\left|T_{i}\right\rangle\left\langle T_{i}\right|$, respectively. ${ }^{12}$ For many experimental EDMR/ODMR studies (the so called continuous-wave experiments), the different description of the observables is not relevant, since these experiments are carried out in the incoherent time regime where only a line-shape analysis of the respective spectra is feasible. However, when coherent effects are studied with pulsed techniques (PEDMR/PODMR), ${ }^{13-17}$ the interpretation of the experiments relies strongly on the proper theoretical description of spin interaction during coherent microwave excitation. ${ }^{8,13}$

An example for the difference between a PEDMR signal and an ESR signal that come from the same spin ensemble are weakly exchange and weakly dipolar coupled distant pair states in the band gap of an arbitrary semiconductor material with weak spin-orbit coupling as described analytically by Boehme and Lips. ${ }^{14}$ Weak spin-spin coupling means that the exchange coupling constant $J$ and dipolar coupling strength $D^{d}$ as defined in Ref. 14 must be much smaller than the difference $\hbar \Delta \omega=\hbar\left(\omega_{a}-\omega_{b}\right)$, which is the difference of the Larmor frequencies $\omega_{a, b}=g_{a, b} \mu_{B} B_{0}$ of the pair partners $a$ and $b$, respectively, times $\hbar$. Note that $\mu_{B}$ represents Bohr's magneton and $B_{0}$ the magnitude of an external magnetic field to which the spin pair is exposed. The requirement of weak spin-spin coupling implies that only pair systems with sufficiently large pair partner distance are considered in this model. Since too large distances and therefore too slow electronic transition rates between the pair partners lead to a loss of the observed signals, the pair distances for which this model is applicable range in the lower $\mathrm{nm}$ range, depending on $\Delta \omega$ given for the pair and the wave functions of the individual pair states. Examples for such systems could be donor-acceptor pairs whose distance is sufficiently large, yet not large enough to make donor-acceptor recombination impossible, ${ }^{18}$ donor deep defect recombination at crystalline silicon surfaces, or equivalently, trap-dangling bond recombination in disordered silicon materials such as amorphous or microcrystalline silicon. ${ }^{8,19}$ Weak spin-orbit coupling is required in order to ensure spin conservation and, therefore, a spin-selection rule. It is fulfilled, for instance, in all known silicon morphologies but also in many organic semiconductor materials. ${ }^{18}$ When the two pair constituents are manipulated identically with a coherent pulse of high field strength ${ }^{23}$ $B_{1} \quad\left(\frac{g \mu_{B} B_{1}}{\hbar}:=\gamma B_{1} \gg \Delta \omega,{ }^{24}\right.$ whereas $\gamma$ is the gyromagnetic ratio), they undergo a simultaneous spin-Rabi oscillation. This can be detected by means of pulse length dependence measurements with both PESR and PEDMR. With PESR, the measurement could be conducted by integration of the free induction decay and would be called a transient nutation experiment, ${ }^{20}$ whereas with PEDMR, the rate relaxation after the coherent excitation would be integrated reflecting the 
pair permutation symmetry within the pairs at the end of the exciting pulse. ${ }^{21}$ While both the PESR and the PEDMR transients would exhibit oscillating signals, the frequency of these oscillations would differ by a factor of 2: The PESR detected nutation frequency $\Omega_{\mathrm{ESR}}=\gamma B_{1}$ would simply represent the Rabi frequency of an uncoupled spin $s=\frac{1}{2}$, whereas the PEDMR measured oscillation would exhibit the frequency at which the identically precessing spins of the two pair partners cross the geometric plane transverse to the field direction of the externally applied magnetic field $B_{0}$ (the $\hat{x}-\hat{y}$ plane), since at these moments the projection of the parallel oriented spins in the $\hat{x}-\hat{y}$ plane onto the spin eigenstates with singlet content will be maximized. Since this plane is passed twice per nutation period, the oscillation of the transition rate is twice as high. Note that this frequency discrepancy of the oscillations between PESR and PEDMR detected transient nutations is changed as the $B_{1}$-field strength becomes weak: When $\gamma B_{1} \ll \Delta \omega$, an ESR excitation will be possible with only one pair partner at the same time. Thus, the maximum singlet content of a pair will be achieved when the spin orientations therein point to opposite directions parallel to the $B_{0}$ field. Hence, for weak $B_{1}$ fields, the nutation frequencies for PESR and PEDMR become equal. In this regard, it shall be noted that we assume that both $J$ and $D^{d}$ and, therefore, the singlet-triplet splitting of the pairs are much smaller than both $\hbar \Delta \omega$ and the strength of the excitation field $\hbar \gamma B_{1}$. A "weak $B_{1}$ field" therefore means $J, D^{d} \ll \hbar \gamma B_{1} \ll \hbar \Delta \omega$.

In the following, a semianalytical study is presented which describes spin-dependent electronic transition rates (e.g., recombination or hopping transport) when the driving forces for Rabi oscillation (under experimental conditions these are typically strong coherent microwave fields) are between a weak $\left(\gamma B_{1} \ll \Delta \omega\right)$ and a strong excitation regime $\left(\gamma B_{1} \gg \Delta \omega\right)$. The goal of this study is to fill the gap between the two analytically derived extremal cases of very weak and very strong excitation as presented by Boehme and Lips ${ }^{14}$ and to describe a general behavior of the nutation frequency reflected by the spin-dependent transition rates for arbitrary $B_{1}$ and $\Delta \omega$ and arbitrary excitation frequencies $\omega$. For a straightforward interpretation of experimental results, ${ }^{22}$ it is of particular interest to understand if the change of Rabi frequency from $\Omega=\Omega_{\mathrm{ESR}}$ to $\Omega=2 \Omega_{\mathrm{ESR}}$ takes place continuously or abruptly.

\section{MODEL FOR SPIN-DEPENDENT RECOMBINATION}

The basis for the results presented in the following is the pair models for spin-dependent recombination and transport as described and discussed in detail in Refs. 14 and 21, respectively. These models are based on the Kaplan-SolomonMott model $^{5}$ under consideration of non-negligible triplet transition probabilities and spin relaxation. For the calculation of the data presented, we strictly follow these models under the assumption of negligible spin-spin interactions. Note that this constraint does not apply to all known experimental systems and will always have to be considered when the results presented in the following are applied to the interpretation of experimental data.
Quantitatively, the models outlined can be represented by an ensemble of spin $s=\frac{1}{2}$ pairs described by the density operator $\hat{\rho}$ as derived in Ref. 14. We define $\hat{H}=\hat{H}_{0}+\hat{H}_{1}(t)$ to be the Hamiltonian of an individual pair with $\hat{H}_{0}=-\frac{1}{2} g_{a} \mu_{B} B_{0} \hat{\sigma}_{\mathbf{z}}^{\mathbf{a}}-\frac{1}{2} g_{b} \mu_{B} B_{0} \hat{\sigma}_{\mathbf{z}}^{\mathbf{b}}$ representing the unperturbed Hamiltonian in the presence of a constant magnetic field $\vec{B}_{0}=B_{0} \hat{\mathbf{z}}$, and $\hat{H}_{1}(t)=-\frac{1}{2} g \mu_{B} B_{1}\left(\hat{\sigma}_{+}^{\mathbf{a}}+\hat{\sigma}_{+}^{\mathbf{b}}\right) e^{-i \omega t}$ is the perturbation with a circularly polarized microwave of angular frequency $\omega$ and strength $B_{1}$. Note the absence of spin-spin coupling in $\hat{H}_{0}$ in contrast to Eq. (5) of Ref. 14. The dynamics of the ensemble of spin pairs can be described by a Liouville equation $\partial_{t} \hat{\rho}=\frac{i}{\hbar}[\hat{\rho}, \hat{H}]^{-}$in which, in contrast to Eq. (1) of Ref. 14, all stochastic terms have been dropped since incoherent processes are considered to be negligible during the applied coherent ESR pulse. Only coherent pulses-these are pulses that are shorter than the fastest incoherent processesare considered in the following. The ensemble of spins as represented by the density operator $\hat{\rho}=\hat{\rho}(t)$ can be expressed by a unitary, time-dependent $4 \times 4$ matrix. Due to the absence of coupling, the Hamiltonian $\hat{H}_{0}$ will be diagonal in the product base with four eigenvalues $\pm \frac{\hbar \omega_{0}}{2}$ and $\pm \frac{\hbar \Delta \omega}{2}$, wherein $\omega_{0}$ and $\Delta \omega$ represent the sum and the difference of the Larmor frequencies $\omega_{a}$ and $\omega_{b}$, respectively.

When a solution for $\hat{\rho}(t)$ is found, the transients of the spin-dependent transition rate

$$
R(t)=\sum_{i=1}^{4} r_{i} \operatorname{Tr}[|i\rangle\langle i| \hat{\rho}(t)]
$$

can be calculated from the projection of the permutation symmetry operators on the ensemble state. Experimentally, a real-time observation of $R(t)$ on typical time scales in the lower ns range is difficult to obtain with PODMR and often impossible with PEDMR due to the insufficient time resolution caused by the long dielectric relaxation times of semiconductors in particular at low temperatures. Hence, transient nutation experiments are typically conducted by means of decay transient measurements as a function of the applied pulse length. ${ }^{15}$ The spin dynamics during the coherent spin excitations are obtained from these pulse length dependence measurements by charge integration $Q(\tau)$, which reveals the permutation symmetry state at the end of the microwave pulse as explained in detail in Ref. 21. Under the given conditions,

$$
Q(\tau) \propto \Delta(\tau)=\frac{\rho_{11}(\tau)-\rho_{11}^{S}}{\operatorname{Tr}\left[\rho^{S}\right]}=\frac{\rho_{44}(\tau)-\rho_{44}^{S}}{\operatorname{Tr}\left[\rho^{S}\right]},
$$

whereas $\rho_{i i}^{S}$ is the steady-state value of the matrix element $\rho_{i i}$ of the density matrix $\hat{\rho}$. Hence, it is $Q(\tau) \propto \Delta(\tau)$, which is the observable calculated and displayed in the following. Note that while $Q(\tau)$ represents a number of charge carriers for PEDMR experiments, the integration of the photoluminescence decay transient in PODMR reveals a number of photons. Nevertheless, in both cases, the observable shall be referred to as $Q(\tau)$ in the following and is always plotted in arbitrary units because of this ambiguity. 


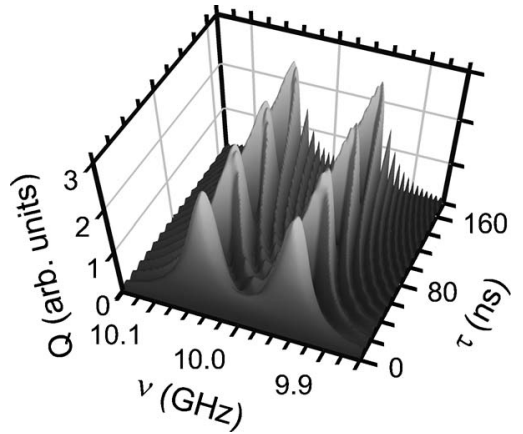

FIG. 1. Plot of the observable $Q$ as a function of the pulse length $\tau$ and the applied microwave frequency $\nu$ for a spin pair with large Larmor separation $\gamma B_{1} \ll \Delta \omega$. The parameters of the simulation were $\frac{\omega_{a}}{2 \pi}=9.95 \mathrm{GHz}$ and $\frac{\omega_{b}}{2 \pi}=10.05 \mathrm{GHz}$ for $0 \leqslant \tau \leqslant 160 \mathrm{~ns}$ and microwave excitation frequencies of $9.86 \leqslant \nu=\frac{\omega}{2 \pi} \leqslant 10.14 \mathrm{GHz}$ as well as a $B_{1}$ field of $\frac{\gamma B_{1}}{2 \pi}=20 \mathrm{MHz}$. One can distinguish the two resonant peaks and recognize the nutation on the pulse length axis.

\section{SIMULATION METHODS AND RESULTS}

When incoherence is negligible, the Hamiltonian $\hat{H}$ can be diagonalized for any given parameter set, and thus the Liouville equation in its time integrated form $\hat{\rho}(\tau)$ $=\exp \left(-\frac{i}{\hbar} \hat{H} \tau\right) \rho^{S} \exp \left(\frac{i}{\hbar} \hat{H} \tau\right)$ can be solved by a simple matrix multiplication in which $\rho^{S}=\frac{1}{2}\left[\left|T_{+}\right\rangle\left\langle T_{+}|+| T_{-}\right\rangle\left\langle T_{-}\right|\right]$is the same initial state as used in Ref. 14. The solution $\hat{\rho}(\tau)$ is referred to as "semianalytic" since this is easy to perform by means of calculation of the eigenvalues and eigenvectors of $\hat{H}$ with any given parameter set, while attempting to solve it fully analytically for arbitrary variables leads to unreasonably lengthy expressions.

Figure 1 displays an example for the time-domain result for $Q(\tau)$ as a function of the Larmor frequency for a spin pair ensemble with a Larmor separation much larger than $\gamma B_{1}$, which is one of the extremal cases discussed in Ref. 14 (Sec. V A 2). One can recognize the two well distinguishable peaks at $\nu=9.95$ and $\nu=10.05 \mathrm{GHz}$ on the Larmor frequency scale determined by the choice of the respective $g$-values of the spin-pair partners as well as the undamped Rabi nutation on the scale of the pulse length $\tau$. Outside of the resonances, the signal intensity drops while the nutation frequency increases. The latter is well known and understood from the expression of the Rabi frequency as introduced in Eq. (28) of Ref. 14. It is due to the spin nutation about the residual $B_{0}$ field $\Delta B_{0}$ in the rotating frame, which increases with the distance of the excitation frequency from the resonance frequency. Figure 1 shows that the relevant information contained in the calculated transients $Q(\tau)$ includes the frequencies as well as the amplitudes of the nutation components. Thus, from the solutions of $Q(\tau)$, the absolute Fourier transform (FT) was calculated in order to analyze the various nutation frequencies contained therein. In contrast to the short 160 ns transient shown in Fig. 1, the time scale for the time-domain simulation and, therefore, the Fourier integration was chosen to be $5 \mu \mathrm{s}$ long. For the parameters used throughout this study, this corresponds to at least 50 Rabi oscillation periods.
Figure 2(a) displays $\Omega=\mathrm{FT}\{Q(\tau)\}$ obtained by semianalytical calculations for spin pairs with four different Larmor separations $\left(\frac{\Delta \omega}{2 \pi}=1,5,20\right.$, and $\left.40 \mathrm{MHz}\right)$ for microwave excitation frequencies of $9.95 \leqslant \nu=\frac{\omega}{2 \pi} \leqslant 10.05 \mathrm{GHZ}$ with a given $B_{1}$ field of $\frac{\gamma B_{1}}{2 \pi}=10 \mathrm{MHz}$. While the plots (a.i) and (a.iv) fulfill the extremal cases of small and large Larmor separation, respectively, the plots (a.ii) and (a.iii) describe two intermediate cases with $\gamma B_{1} \approx \Delta \omega$. Note that the scaling of the color code was normalized to the maximum for each graph in order to achieve sufficient contrast. In order to be able to compare the four different cases more easily, the Rabi components $\operatorname{FT}\{Q(\tau)\}$ at the resonance frequency of the two spin partners are plotted in the respective graphs (red curves) as conventional two-dimensional plots. Here, the chosen scaling is equal for all graphs. The four cases displayed in Fig. 2(a) confirm the hyperbolic increase of the Rabi frequency $\Omega=\sqrt{\left(\gamma B_{1}\right)^{2}+\left(\omega-\omega_{a, b}\right)^{2}}$ as the microwave frequency is shifted out of resonance. One can deduce from the twodimensional inset plots of Fig. 2(a.i) and (a.iv) that the onresonance cases show only one frequency component for the two extremal cases, namely $\Omega=\gamma B_{1}$ for large Larmor separation and $\Omega=2 \gamma B_{1}$ for small Larmor separation. This confirms the analytical results of Ref. 14. Outside of the resonances $\left(\omega \neq \omega_{a, b}\right)$, the oscillation splits into two components for the extremal cases, and in the general, intermediate cases there are up to four different nutation frequencies.

In order to illustrate the transition from a single $\Omega=\gamma B_{1}$ to a single $\Omega=2 \gamma B_{1}$ frequency component with decreasing Larmor separation (or equivalently, for an increasing microwave field $B_{1}$ ), a plot of $\Omega=\operatorname{FT}\{Q(\tau)\}$ versus the ratio $\frac{\Delta \omega}{\gamma B_{1}}$ on a logarithmic scale around $\frac{\Delta \omega}{\gamma B_{1}}=1$ is shown in Fig. 3 for the one-spin on-resonant cases $\omega=\omega_{a, b}$ (a) and the average frequency case $\omega=\omega^{\prime}=\frac{1}{2}\left(\omega_{a}+\omega_{b}\right)(\mathrm{b})$. Again, for the extremal cases of $\frac{\Delta \omega}{\gamma B_{1}} \ll 1$ and $\frac{\Delta \omega}{\gamma B_{1}} \gg 1$, the plots confirm the known results for small and large Larmor separation described above. In Fig. 3(a), one can see for $\frac{\Delta \omega}{\gamma B_{1}} \ll 1\left(\log \left[\frac{\Delta \omega}{\gamma B_{1}}\right]<0\right)$ that there is only one component with $\Omega=2 \gamma B_{1}$. As $\frac{\Delta \omega}{\gamma B_{1}}$ increases and approaches $1\left(\log \left[\frac{\Delta \omega}{\gamma B_{1}}\right]\right.$ approaches 0$)$, this component gradually becomes weaker and its frequency increases. This behavior can be understood by the fact that in Fig. 3(a), $\omega$ is always equal to one of the pair partner resonances. When $\Delta \omega$ becomes larger, $\omega \neq \omega^{\prime}$ and thus an increase of the observed nutation frequency takes place. When $\frac{\Delta \omega}{\gamma B_{1}} \approx 1 \quad\left(\log \left[\frac{\Delta \omega}{\gamma B_{1}}\right] \approx 0\right)$, two new nutation components become visible: (i) A low-frequency component that generally is hard to separate from any given offset in the function $Q(\tau)$, and (ii) one component with $\Omega=\gamma B_{1}$. The magnitude of the latter rises from very small values for $\frac{\Delta \omega}{\gamma B_{1}} \ll 1$ and increases asymptotically to a maximum value for $\frac{\Delta \omega}{\gamma B_{1}} \gg 1$, whereas the two other components vanish. A complementary view on these changes is given by Fig. 3(b), where the Rabi components for an excitation frequency $\omega=\omega^{\prime}$ are plotted versus $\frac{\Delta \omega}{\gamma B_{1}}$. For $\frac{\Delta \omega}{\gamma B_{1}} \ll 1$, plots (a) and (b) agree since they represent the same physical situation. When $\frac{\Delta \omega}{\gamma B_{1}}$ increases and $\omega_{a, b} \neq \omega^{\prime}=\omega$, no low-frequency component becomes visible. The $\Omega=\gamma B_{1}$ component, which also becomes visible, will increase proportionally to $\Delta \omega$ since it is off-resonant to the applied microwave frequency $\omega$. 

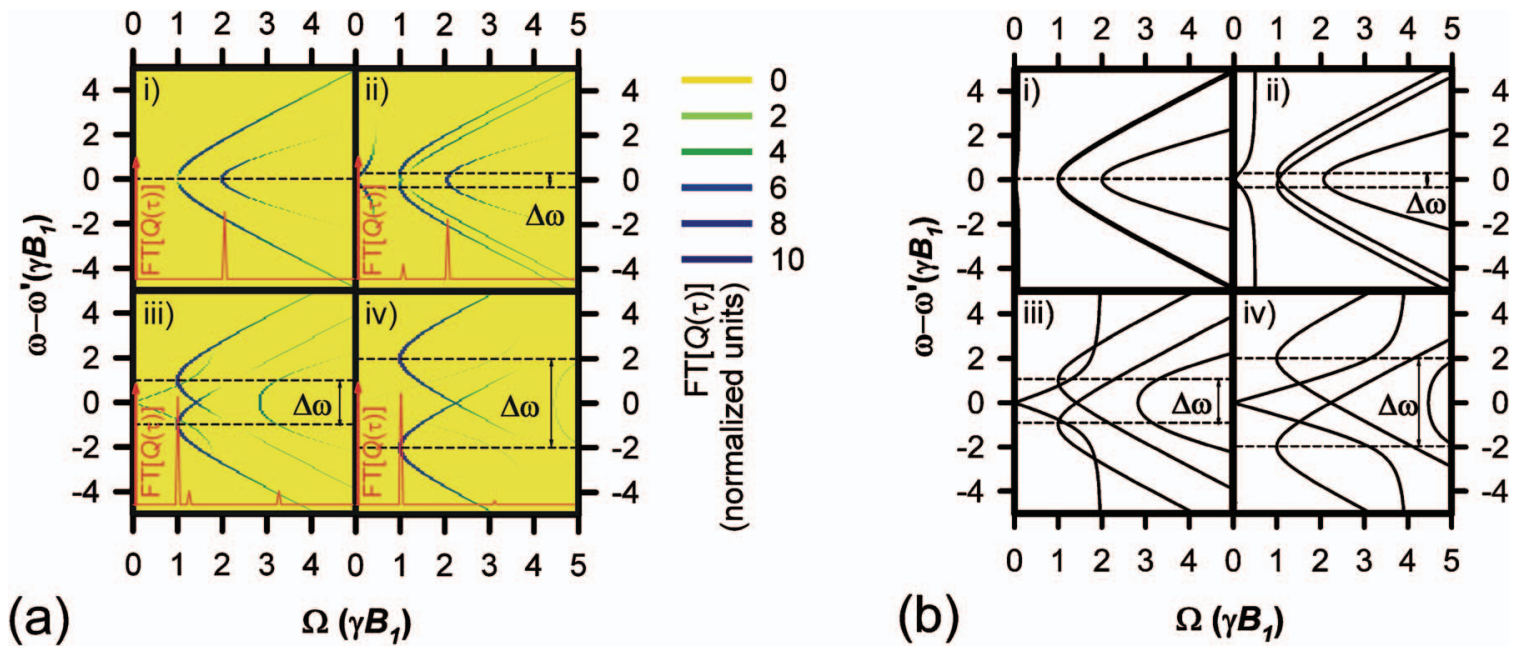

FIG. 2. (Color) (a) Three-dimensional color plots of the semianalytically calculated $\Omega=\mathrm{FT}\{Q(\tau)\}$ as a function of the excitation frequency $\omega$ scaled in units of $\gamma B_{1}$ as the difference between $\omega$ and the average of the Larmor frequencies of the two pair partners $\omega^{\prime}$ $=\frac{1}{2}\left(\omega_{a}+\omega_{b}\right)$ and the Rabi-nutation frequency $\Omega$ in units of $\gamma B_{1}$. For all four plots (a.i) to (a.iv), $\frac{\gamma}{2 \pi} B_{1}=10 \mathrm{MHz}$ and $\frac{\omega^{\prime}}{2 \pi}=10 \mathrm{GHz}$. From plot (a.i) to (a.iv), the Larmor separation increases: (a.i), $\frac{\Delta \omega}{2 \pi}=1 \mathrm{MHz}$; (a.ii), $\frac{\Delta \omega}{2 \pi}=5 \mathrm{MHz}$; (a.iii), $\frac{\frac{\Delta \omega}{2 \pi}}{2 \pi}=20 \mathrm{MHz}$; (a.iv), $\frac{\Delta \omega}{2 \pi}=40 \mathrm{MHz}$. The twodimensional inset plots display the data of the three-dimensional plot at the Larmor frequency slices indicated by the dashed lines. (b) The plot of the Rabi-nutation frequencies as obtained from Eqs. (3) and (4) for the same parameters as used for the calculated results in (a). A comparison with the frequencies therein shows an excellent agreement.

\section{DISCUSSION}

The simulation of spin-Rabi oscillation as observed by PEDMR/PODMR reveals that the doubling of the nutation frequency $\Omega=\gamma B_{1}$ to $\Omega=2 \gamma B_{1}$ with decreasing Larmor separation $\left(\frac{\Delta \omega}{\gamma B_{1}}\right)$ is abrupt, which means that there is no continuous increase of the oscillation frequency. Instead, only the magnitudes of the various components change in the intermediate Larmor separation regime about $\frac{\Delta \omega}{\gamma B_{1}} \approx 1$. Here, four nutation components become visible, which can all become significant at the same time. Mathematically, it is obvious that the pairs consisting of two $s=\frac{1}{2}$ particles with four eigenstates will reveal four eigenfrequencies for any given general set of parameters. We interpret this behavior for the general case as the interplay of the two one-spin systems and the one two-spin system by which the spin pairs are described in the two extremal cases discussed above. The results of the analytical derivation given in Ref. 14 showed that the most sig-

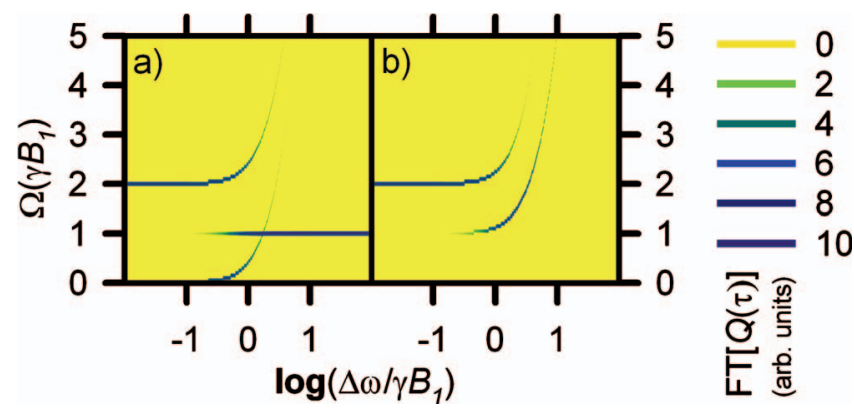

FIG. 3. (Color) Color plot of the Rabi-frequency components $\Omega=\mathrm{FT}\{Q(\tau)\}$ as function of $\log \left(\frac{\Delta \omega}{\gamma B_{1}}\right)$ for an excitation frequency (a) $\omega=\omega_{a, b}$ on resonance with one of the pair partners and (b) $\omega=\omega^{\prime}$ on resonance with the average $\omega^{\prime}$ of the pair partners Larmor frequencies. nificant qualitative change that takes place when the Larmor separation changes from infinity to zero is that a transition from a one-spin to a two-spin system occurs. For the onespin system, the spin in resonance (note that here, only one spin can be in resonance) fully determines the oscillation of the pair permutation symmetry, whereas for the two-spin system, the permutation symmetry is determined by the relative movements and spin orientations within the pair ensemble. For large Larmor separation, at least one spin will remain fixed in its initial state, typically an eigenstate with polarization parallel to the $B_{0}$ axis. As the excitation frequency is changed, the system becomes off-resonant, and two one-spin contributions

$$
\Omega_{a, b}=\sqrt{\left(\gamma B_{1}\right)^{2}+\left(\omega-\omega_{a, b}\right)^{2}}
$$

appear where the frequency of one increases whereas the frequency of the other decreases at the same time. The pair still behaves like two individual one-spin systems. For small Larmor separation, there are always two spins in motion as long as the system is on resonance (there is then only one resonance line observed). Hence, since the relative spin motion of the two spins within the pair will now determine the oscillation of the electronic rate transition, the beat frequencies

$$
\Omega_{p, n}=\Omega_{a} \pm \Omega_{b}
$$

of the two one-spin nutation frequencies can be expected. Here, $\Omega_{p}$ and $\Omega_{n}$ stand for parallel and antiparallel orientations, respectively. Figure 2(b) displays four plots of the nutation frequencies obtained with the simple terms given in Eqs. (3) and (4) for the same parameter sets used for the simulation results displayed in Fig. 2(a). While this purely phenomenological description of the nutation frequencies cannot account for the intensities of the nutation 
components-this is the reason why there is no gray scale gradient in Fig. 2(b) - it nevertheless shows that there is an excellent agreement of Eqs. (3) and (4) with the semianalytically calculated frequency patterns displayed in Fig. 2(b).

While simple quantitative predictions can be made for the nutation frequencies, there are no straightforward formulas for the prediction of the nutation amplitudes. As shown in Fig. 2(a), the intensities of the different nutation components can have quite complex microwave frequency $(\omega)$ dependencies. Well separated lines (as in the case of large Larmor separation) exhibit Lorentzian line shapes determined by the $B_{1}$ field due to the power broadening as described in Ref. 14 . For intermediate cases, the $\omega$ dependence becomes much more complex and a given nutation component can exhibit a maximum at its corresponding resonance but also a local minimum at the resonance of a noncorresponding transition. An example for this behavior is the data of Fig. 2(a.iii). At a frequency of $\omega-\omega^{\prime}= \pm \gamma B_{1}$, one can recognize both a maximum of the nutation component at $\Omega=\gamma B_{1}$ but also a minimum of the respective other nutation component at $\Omega$ $\approx 2 \gamma B_{1}$. Qualitatively, this behavior can be interpreted by consideration of a four-level system. Any of the four levels can undergo first-order transitions into two different states. For excitations that are out of resonance with both transitions, the transition probability is small but may not be negligible in the vicinity of the resonances. However, when one transition is induced resonantly, the transition into the nonresonantly excited state is quenched at the same time and the intensity of its corresponding nutation component is quenched. Note that in spite of this qualitative interpretation, quantitative predictions have to be made by means of the simulation methods described above.

\section{SUMMARY AND CONCLUSIONS}

In summary, the response of charge-carrier transport and recombination rates through localized electronic states in semiconductors to a coherent manipulation by magnetic resonance were simulated as they would be expected in PEDMR/PODMR experiments. The transient response was calculated with the spin excitation present for different excitation fields and frequencies as well as different Larmor separations within the pairs. It was assumed that exchange and dipolar interaction are weak and incoherence due to the electronic transitions or spin relaxation is negligible. The presented data were obtained by a semianalytical simulation method. The results show that four qualitatively distinguishable nutation processes influence the oscillation of the transition rates, which reduce to one significant contribution in the cases of large and small Larmor separations. Simple empirical equations for the calculation of these nutation frequencies have been obtained that match the simulated data excellently, and a qualitative picture for the interpretation of the nutation intensities has been discussed. The presence of the four nutation processes implies that changing the Larmor separation or the applied excitation field leads to an abrupt and not continuous change of the observed nutation frequencies.
${ }^{1}$ B. C. Cavenett, Adv. Phys. 30, 475 (1981).

${ }^{2}$ M. Stutzmann, M. S. Brandt, and M. W. Bayerl, J. Non-Cryst. Solids 266-269, 1 (2000).

${ }^{3}$ M. Xia, I. Martin, E. Yablonovitch, and H. W. Jiang, Nature (London) 430, 435 (2004).

${ }^{4}$ F. Jelezko, T. Gaebel, I. Popa, A. Gruber, and J. Wrachtrup, Phys. Rev. Lett. 92, 076401 (2004).

${ }^{5}$ D. Kaplan, I. Solomon, and N. F. Mott, J. Phys. (Paris), Lett. 39, L51 (1978).

${ }^{6}$ D. J. Lepine, Phys. Rev. B 6, 436 (1972).

${ }^{7}$ F. C. Rong, W. R. Buchwald, E. H. Poindexter, W. L. Warren, and D. J. Keeble, Solid-State Electron. 34, 835 (1991).

${ }^{8}$ C. Boehme and K. Lips, Phys. Status Solidi C 1, 1255 (2004).

${ }^{9}$ F. Friedrich, C. Boehme, and K. Lips, J. Appl. Phys. 97, 056101 (2005).

${ }^{10}$ T. Eickelkamp, S. Roth, and M. Mehring, Mol. Phys. 95, 967 (1998).

${ }^{11}$ K. Lips and W. Fuhs, J. Appl. Phys. 74, 3993 (1993).

${ }^{12}$ R. Haberkorn and W. Dietz, Solid State Commun. 35, 505 (1980).

${ }^{13}$ C. Boehme and K. Lips, Phys. Rev. Lett. 91, 246603 (2003).

${ }^{14}$ C. Boehme and K. Lips, Phys. Rev. B 68, 245105 (2003).

${ }^{15}$ D. J. Gravesteijn and M. Glasbeek, Phys. Rev. B 19, 5549 (1979).

${ }^{16}$ J. Wrachtrup, C. von Borczyskowski, J. Bernard, M. Orrit, and R. Brown, Nature (London) 363, 244 (1993).

${ }^{17}$ J. Köhler, J. A. J. M. Disselhorst, M. C. J. M. Donckers, E. J. J.
Groenen, J. Schmidt, and W. E. Moerner, Nature (London) 363, 242 (1993).

${ }^{18}$ J.-M. Spaeth and H. Overhof, Point Defects in Semiconductors and Insulators (Springer, Berlin, 2002).

${ }^{19}$ K. Lips, C. Boehme, and T. Ehara, J. Optoelectron. Adv. Mater. 7, 13 (2004).

${ }^{20}$ A. Schweiger and G. Jeschke, Principles of Pulse Electron Paramagnetic Resonance (Oxford University Press, Oxford, 2001).

${ }^{21}$ C. Boehme and K. Lips, in Charge Transport in Disordered Solids with Applications in Electronics, edited by S. Baranovski (John Wiley and Sons, Ltd., Chichester, England, 2006), Chap. 5, pp. 179-219.

${ }^{22}$ C. Boehme and K. Lips, Physica B 376-377, 930 (2006).

${ }^{23}$ Note that throughout this work, the value $B_{1}$ for the strength of the resonant microwave field is defined as the strength of the constant magnetic field in the rotating frame, which represents also the amplitude of a circular polarized field in the laboratory frame. This is in contrast to the definition of the $B_{1}$ field in Ref. 14 , which is linearly polarized along the $\hat{x}$ axis

${ }^{24}$ Note that $g$ may be either one of the two Landé factors $g_{a}$ or $g_{b}$ or it may even be assumed to be the free-electron Landé factors $g=g_{e}$ since the differences between these values in comparison to their magnitude is negligible here and in all other terms throughout this study where $g$ appears. For $g=g_{e}, \frac{g \mu_{B}}{\hbar}:=\gamma$ is the gyromagnetic ratio. 